\title{
New Results on the Pseudorandomness of Some Blockcipher Constructions
}

\author{
Henri Gilbert and Marine Minier \\ France Télécom R\&D \\ 38-40, rue du Général Leclerc \\ 92794 Issy les Moulineaux Cedex 9 \\ France
}

\begin{abstract}
In this paper, we describe new results on the security, in the Luby-Rackoff paradigm, of two modified Feistel constructions, namely the L-scheme, a construction used at various levels of the MISTY blockcipher which allows to derive a $2 n$-bit permutation from several $n$-bit permutations, and a slightly different construction named the R-scheme. We obtain pseudorandomness and super-pseudorandomness proofs for Lschemes and R-schemes with a sufficient number of rounds, which extend the pseudorandomness and non superpseudorandomness results on the 4-round L-scheme previously established by Sugita [Su96] and Sakurai et al. [Sa97]. In particular, we show that unlike the 3-round L-scheme, the 3-round R-scheme is pseudorandom, and that both the 5-round $\mathrm{L}$ scheme and the 5-round $\mathrm{R}$ scheme are super pseudorandom (whereas the 4 round versions of both schemes are not super pseudorandom). The security bounds obtained here are close to those established by Luby and Rackoff for the three round version of the original Feistel scheme.
\end{abstract}

\section{Introduction}

A key dependent cryptographic function such as a blockcipher can be viewed as a random function associated with a randomly selected key value. It is generally defined using a recursive construction process. Each step of the recursion consists of deriving a random function (or permutation) $f$ from $r$ previously defined random functions (or permutations) $f_{1}, . ., f_{r}$, and can be represented by a relation of the form $f=\Phi\left(f_{1}, . ., f_{r}\right)$. The most studied example so far is the $f=\Psi\left(f_{1}, . ., f_{r}\right) r$-round Feistel construction, which allows to derive a $2 n$-bit to $2 n$-bit random permutation from $r n$-bit to $n$-bit functions. But there exist other well known constructions such as for instance Massey and Lai's alternative to the Feistel scheme used in IDEA [La90] and the constructions allowing to deduce a $2 n$-bit permutation from several $n$-bit permutations used in Matsui's MISTY blockcipher [Ma93].

The strongest security requirement one can put on a $f$ random function or permutation representing a key dependent cryptographic function is (informally speaking) that $f$ be undistinguishable with a non negligible success probability from a perfect random function $f^{*}$ or permutation $c^{*}$, even if a probabilistic 
testing algorithm $A$ of unlimited power is used for that purpose and if the $q$ number of adaptively chosen queries of $A$ to the random instance of $f$ or $f^{*}$ to be tested is large.

It is generally not possible to prove undistiguishability properties for "real life" cryptologic random function $f$ and large $q$ numbers of queries, because this would require a far too long key length. However, it is often possible to prove or disprove that if a random function $f$ encountered at a given level of a cryptologic function construction is related to random functions encountered at the lower recursion level by a relation of the form $f=\Phi\left(f_{1}, . ., f_{r}\right)$, then if we replace the actual $f_{1}$ to $f_{r}$ random functions of the cipher by independent perfect random functions or permutations $f_{1}^{*}$ to $f_{r}^{*}$ (or, in a more sophisticated version of the same approach, by $f_{1}^{\prime}$ to $f_{r}^{\prime}$ functions which are sufficiently undistinguishable from $f_{1}^{*}$ to $f_{r}^{*}$ ), the resulting modified $f$ random function is undistinguishable from a random function (or permutation). This provides a useful method for assessing the soundness of blockcipher constructions. For instance, in the case of a three-round Feistel construction, a well known theorem first proved by Luby and Rackoff [Lu88] provides upper bounds on the $\left|p-p^{*}\right|$ advantage of any $A$ testing algorithm in distinguishing the $f=\Psi\left(f_{1}^{*}, f_{2}^{*}, f_{3}^{*}\right) 2 n$-bit random permutation deduced from three independent ideal random functions $f_{1}^{*}, f_{2}^{*}$ and $f_{3}^{*}$ from a $2 n$-bit perfect random permutation $c^{*}$ with $q$ adaptively chosen queries to the tested instance of $f$ or $f^{*}$. This advantage is bounded over by $\frac{q^{2}}{2^{n}}$.

The research on pseudorandomness properties of cryptographic constructions initiated Luby and Rackoff's seminal paper [Lu88] has represented a very active research study for the last decade. Just to mention a few examples, Zheng, Matsumoto and Imai and later on Sugita and Sakurai et al. investigated generalised Feistel constructions [Zh89],[Su96],[Su97], Patarin explicited the link between the best advantage of a $q$-queries distinguisher and the $q$-ary transition probabilities associated with $f$ and proved undistinguihability bounds for numerous r-round Feistel constructions [Pa91], Maurer showed how to generalise undistinguishability results related to perfect random functions to undistinguishability results related to nearly perfect random functions (e.g. locally random functions)[Ma92], Bellare, Kilian, Rogaway et al. [Be94] investigated the application of similar techniques to modes of operation such as CBC MACs, Aiello and al. proved undistiguihability results on some parallelizable alternatives to the Feistel construction [Ai96], Vaudenay embedded techniques for deriving undistinguishability bounds into a broader framework he named the decorrelation theory, and applied bounds provided by decorrelation techniques to proving the resistance of actual ciphers, e.g. DFC, against differential and linear cryptanalysis.

In this paper, we describe new results on the security of some blockcipher constructions in the above described paradigm, i.e. we investigate some $f=\Phi\left(f_{1}, . ., f_{k}\right)$ constructions and upper bound the probability of distinguishing $f$ from a perfect random function when $\Phi$ is applied to perfect random functions $f_{i}^{*}$ or to perfect random permutations $c_{i}^{*}$. We consider alternatives to the Feistel construction allowing to derive a $2 n$-bit permutation from several $n$-bit permutations, namely the so-called L-scheme and R-scheme constructions. The L-type 
construction is used for instance at various levels of the construction of Matsui and al. Misty blockcipher [Ma93], as well as in the Kasumi variant of Misty recently adopted as the standard blockcipher for encryption and integrity protection in third generation mobile systems [Ka]. We obtain pseudorandomness and superpseudorandomness proofs for L-scheme and R-scheme constructions with a sufficient number of rounds, which extend the results on the pseudo randomness of the 4-round L-scheme previously established by Sugita [Su96] and Sakurai et al. [Sa97]. In particular, we show that unlike the 3-round L scheme, the 3-round $\mathrm{R}$ scheme is pseudorandom, and that both the 5-round $\mathrm{L}$ scheme and the 5-round $\mathrm{R}$ scheme are super pseudorandom (whereas the 4 round versions of both schemes are not super pseudorandom).

This paper organised as follows: Section 2 introduces basic definitions and useful general results on random functions and techniques for proving that two random functions are undistiguishable. Section 3 describes the $\mathrm{R}$ and $\mathrm{L}$ schemes. Sections 4 and 5 present our results on the pseudo-randomness and the superpseudorandomness of the L-scheme and the R-scheme respectively, for various numbers of rounds, and Section 6 concludes the paper.

\section{Preliminaries}

\subsection{Notation}

Through this paper we are using the following notation: $I_{n}$ denotes the $\{0,1\}^{n}$ set. $F_{n, m}$ denotes the $I_{n}{ }^{I_{m}}$ set of functions from $I_{n}$ into $I_{m}$ : thus $\left|F_{n, m}\right|=2^{m .2^{n}}$. $F_{n}$ denotes the $F_{n, n}$ set: thus $\left|F_{n}\right|=2^{n .2^{n}} . P_{n}$ denotes the set of permutations on $I_{n}$ : thus $\left|P_{n}\right|=2^{n}$ !.

\subsection{Random Functions}

A random function of $F_{n, m}$ is defined as a random variable $f$ of $F_{n, m}$, and can be viewed as a probability distribution $(\operatorname{Pr}[f=\varphi])_{\varphi \in F_{n, m}}$ over $F_{n, m}$, or equivalently as a $\left(f_{\omega}\right)_{\omega \in \Omega}$ family of $F_{n, m}$ elements. In particular:

- A $n$-bit to $m$-bit key dependent cryptographic function is determined by a randomly selected key value $K \in \mathcal{K}$, and can thus be represented by the random function $f=\left(f_{K}\right)_{K \in \mathcal{K}}$ of $F_{n, m}$.

- A cryptographic construction of the form $f=\Phi\left(f_{1}, f_{2}, . ., f_{r}\right)$ can be viewed as a random function of $F_{n, m}$ determined by $r$ random functions $f_{i} \in$ $F_{n_{i}, m_{i}}, i=1 . . r$

Definition 1. We define a perfect random function $f^{*}$ of $F_{n, m}$ as a uniformly drawn element of $F_{n, m}$. In other words, $f^{*}$ is associated with the uniform probability distribution over $F_{n, m}$. We define a $c^{*}$ perfect random permutation on $I_{n}$ as a uniformly drawn element of $P_{n}$. In other words, $c^{*}$ is associated with the uniform probability distribution over $P_{n}$. 
Definition 2. (t-ary transition probabilities associated with $f$ ). Given a random function $f$ of $F_{n, m}$, we define the $\operatorname{Pr}[x \stackrel{f}{\mapsto} y]$ transition probability associated with a $x$ t-uple of $I_{n}$ inputs and a $y$ t-uple of $I_{m}$ outputs as

$\operatorname{Pr}[x \stackrel{f}{\mapsto} y]=\operatorname{Pr}\left[f\left(x_{1}\right)=y_{1} \wedge f\left(x_{2}\right)=y_{2} \wedge \ldots \wedge f\left(x_{t}\right)=y_{t}\right]$
$=\operatorname{Pr}_{\omega \in \Omega}\left[f_{\omega}\left(x_{1}\right)=y_{1} \wedge f_{\omega}\left(x_{2}\right)=y_{2} \wedge \ldots \wedge f_{\omega}\left(x_{t}\right)=y_{t}\right]$

In the sequel we will use the following simple properties:

Property 1 If $f^{*}$ is a perfect random function $F_{n, m}$ and if $x=\left(x_{1}, \ldots, x_{t}\right)$ is a t-uple of pairwise distinct $I_{n}$ values and if $y$ is any $t$-uple of $I_{m}$ values, then $\operatorname{Pr}\left[x \stackrel{f^{*}}{\mapsto} y\right]=\frac{1}{\left|I_{m}\right|^{t}}=2^{-m . t}$

Property 2 Let $c^{*}$ be a perfect random permutation on $I_{n}$. If $x=\left(x_{1}, \ldots, x_{t}\right)$ is a t-uple of pairwise distinct $I_{n}$ values $y=\left(y_{1}, \ldots, y_{t}\right)$ is a $t$-uple of pairwise distinct $I_{n}$ values then $\operatorname{Pr}\left[x \stackrel{c^{*}}{\mapsto} y\right]=\left(I_{n}-t\right) ! /|\operatorname{In}| !=\frac{\left(2^{n}-t\right) !}{\left(2^{n}\right) !}$

Property 3 Let $c^{*}$ be a perfect random permutation on $I_{n}$. If $x$ and $x^{\prime}$ are two distinct elements of $I_{n}$ and $\delta$ is a given value of $I_{n}$ then $\operatorname{Pr}\left[c^{*}(x) \oplus c^{*}\left(x^{\prime}\right)=\delta\right] \leq$ $\frac{2}{2^{n}}$.

Proof: $\operatorname{Pr}\left[c^{*}(x) \oplus c^{*}\left(x^{\prime}\right)=0\right]=0$ since $x \neq x^{\prime}$. If $\delta \neq 0, \operatorname{Pr}\left[c^{*}(x) \oplus c^{*}\left(x^{\prime}\right)=\delta\right]=$ $\frac{2^{n} \cdot 2^{n-2} \cdots 1}{2^{n} !}=\frac{1}{2^{n}-1} \leq \frac{2}{2^{n}}$. So, $\operatorname{Pr}\left[c^{*}(x) \oplus c^{*}\left(x^{\prime}\right)=\delta\right] \leq \frac{2}{2^{n}}$.

\subsection{Distinguishing Two Random Functions}

In proofs of security such as the one presented here, we want to upper bound the probability of any algorithm to distinguish whether a given fixed function $\varphi$ is an instance of a random function $f=\Phi\left(f_{1}^{*}, f_{2}^{*}, \ldots, f_{r}^{*}\right)$ of $F_{n, m}$ or an instance of the perfect random function $f^{*}$, using less than $q$ queries to $\varphi$.

Let $A$ be any distinguishing algorithm of unlimited power that, when input with a function $\varphi$ of $F_{n, m}$ (which can be modeled as an " oracle tape " in the probabilistic Turing Machine associated with A) selects a fixed number $q$ of distinct chosen or adaptively chosen input values $X_{i}$ (the queries), obtains the $q$ corresponding output values $Y_{i}=f\left(X_{i}\right)$, and based on these results outputs 0 or 1 . Denote by $p$ (resp by $p^{*}$ ) the probability for $A$ to answer 1 when fed with a random instance of $f$ (resp of $f^{*}$ ). We want to find upper bounds on the $A d v_{A}\left(f, f^{*}\right)=\left|p-p^{*}\right|$ advantage of $A$ in distinguishing $f$ from $f^{*}$ in $q$ queries.

As first noticed by Patarin [Pa91], the best $A d v_{A}\left(f, f^{*}\right)$ advantage of any A distinguishing algorithm for distinguishing $f$ from $f^{*}$ is entirely determined by the $\operatorname{Pr}[x \stackrel{f}{\mapsto} y] q$-ary transition probabilities associated with each $X=$ $\left(X_{1}, \cdots, X_{q}\right)$ q-uple of pairwise distinct $I_{n}$ values and each $Y=\left(Y_{1}, \cdots, Y_{q}\right)$ $q$-uple of $I_{m}$ values. The following Theorem, which was first proved in [Pa91], and equivalent versions of which can be found in [Va99], is a very useful tool for deriving establishing upper bounds on the $A d v_{A}\left(f, f^{*}\right)$ based on properties of the $\operatorname{Pr}[x \stackrel{f}{\mapsto} y] q$-ary transition probabilities. 
Theorem 1 Let $f$ be a random function of $F_{n, m}$ and $f^{*}$ a perfect random function representing a uniformly drawn random element of $F_{n, m}$. Let $q$ be an integer. Denote by $\mathcal{X}$ the $I_{n}{ }^{q}$ set of all $X=\left(X_{1}, \cdots, X_{q}\right)$ q-tuples of pairwise distinct elements. If there exists a $\mathcal{Y}$ subset of $I_{m}{ }^{q}$ and two positive real numbers $\epsilon_{1}$ and $\epsilon_{2}$ such that

1) $|\mathcal{Y}|>\left(1-\epsilon_{1}\right) \cdot\left|I_{m}\right|^{q}$ (i)

2) $\forall X \in \mathcal{X} \forall Y \in \mathcal{Y} \operatorname{Pr}[X \stackrel{f}{\mapsto} Y] \geq\left(1-\epsilon_{2}\right) \cdot \frac{1}{\left|I_{m}\right|^{q}}$ (ii)

then for any $A$ distinguishing using q queries

$$
A d v_{A}\left(f, f^{*}\right) \leq \epsilon_{1}+\epsilon_{2}
$$

In order to improve the selfreadability of this paper, a short proof of Theorem 1 is provided in appendix at the end of this paper.

\section{Description of the L- and R-Schemes}

We now describe two simple variants of the Feistel scheme, that we propose to name L-scheme and R-scheme, following the terminology proposed by Kaneko and al. in their paper on the provable security against differential and linear cryptanalysis of generalised Feistel ciphers [Ka97].

The L-scheme and R-scheme both allow to derive a 2 n-bit to 2 n-bit permutation from several $n$-bit to $\mathrm{n}$ bit permutations (not only n-bit to n-bit functions as in the Feistel scheme), using only one n-bit to $\mathrm{n}$ bit permutation per round.

The 1-round L-scheme is depicted in Figure 1. It transforms a $c_{1}$ permutation of $I_{n}$ into the $\psi_{L}\left(c_{1}\right)$ permutation of $I_{2 n}$ defined by

$$
\psi_{L}\left(c_{1}\right)\left(x^{1}, x^{0}\right)=\left(x^{0}, c_{1}\left(x^{1}\right) \oplus x^{0}\right)
$$

The extension to $r$ rounds is straightforward: the $r$-round L-scheme transforms $r I_{n}$ permutations $c_{1}$ to $c_{r}$ into the $I_{2 n}$ permutation defined by

$$
\psi_{L}\left(c_{1}, c_{2}, \ldots, c_{r}\right)=\psi_{L}\left(c_{r}\right) \circ \cdots \circ \psi_{L}\left(c_{1}\right)
$$

The L-scheme is used at several levels of the construction of the MISTY and KASUMI ciphers, namely the derivation of the so-called FI and FO functions, and also the upper level of the construction in the case of MISTY2. One remarkable feature of the r-round L-scheme is that two $c_{i}$ permutations can be processed in paralell.

The 1-round R-scheme is depicted in Figure 1 too. It transforms a $c 1$ permutation of $I_{n}$ into the $\psi_{R}\left(c_{1}\right)$ permutation of $I_{2 n}$ defined by

$$
\psi_{R}\left(c_{1}\right)\left(x^{1}, x^{0}\right)=\left(c_{1}\left(x^{1}\right) \oplus x^{0}, c_{1}\left(x^{1}\right)\right)
$$

The r-round R-scheme transforms $r I_{n}$ permutations $c_{1}$ to $c_{r}$ into the $I_{2 n}$ permutation defined by $\psi_{R}\left(c_{1}, c_{2}, \ldots, c_{r}\right)=\psi_{R}\left(c_{r}\right) \circ \cdots \circ \psi_{R}\left(c_{1}\right)$. 

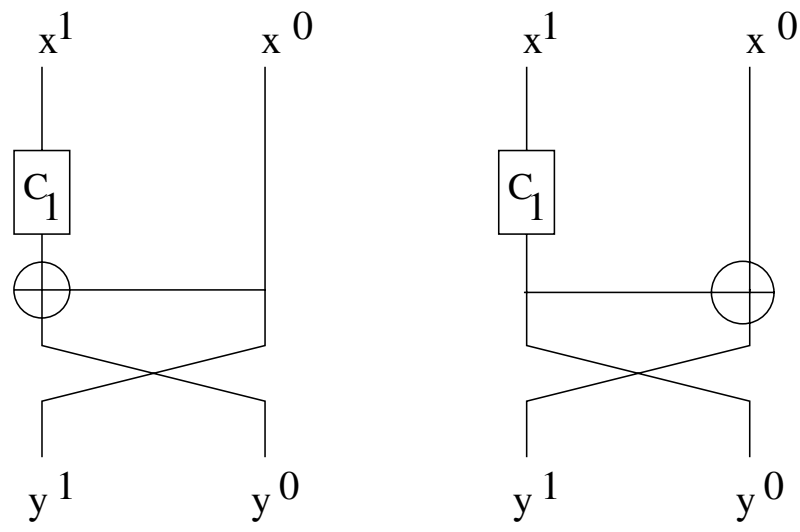

Fig. 1. L-scheme one round at left and R-scheme one round at right

In the sequel, we will several times consider the slightly simplified versions $\psi_{L}^{\prime}\left(c_{1}, c_{2}, \ldots, c_{r}\right)$ and $\psi_{R}^{\prime}\left(c_{1}, c_{2}, \ldots, c_{r}\right)$ of $\psi_{L}\left(c_{1}, c_{2}, \ldots, c_{r}\right)$ and $\psi_{R}\left(c_{1}, c_{2}, \ldots, c_{r}\right)$ obtained by omitting the XOR operation and the exchange of the left and right halves in the final round. We will sometimes analyse such simplified variants, whose pseudorandomness properties are obviously the same as those of the full $r$-round $\mathrm{L}$ or $\mathrm{R}$ scheme from which they are derived, instead of the full r-round $\mathrm{L}$ or $\mathrm{R}$ scheme, in order to simplify some discussions. We can notice that the $\psi_{R}^{\prime}\left(c_{1}, c_{2}, \ldots, c_{r}\right)$ and $\psi_{L}^{\prime}\left(c_{r}^{-1}, c_{r-1}^{-1}, \ldots, c_{1}^{-1}\right)$ permutations are inverse of each other. This remark will be useful when it comes to analysing the super pseudorandomness properties of the $\mathrm{L}$ and $\mathrm{R}$ schemes.

Through the two next Sections, especially in proofs, we are using the following additional notation:

- $I$ is an abreviation for the $\left(I_{n}\right)^{q}$ set.

$\left.-I^{\neq}=\left(\left(I_{n}\right)^{q}\right)\right)^{\neq}$denotes the subset of $\left(I_{n}\right)^{q}$ consisting of all the q-tuples of pairwise distinct $I_{n}$ values and $I^{=}=\left(I_{n}\right)^{q} \backslash I^{\neq}$.

$-\mathcal{X}$ denotes the subset of $\left(I_{2 n}\right)^{q}$ consisting of all $\left(x_{1}, \cdots, x_{q}\right) q$-tuples of pairwise distinct $I_{2 n}$ values.

$-\mathcal{Y}$ will denote a subset of $\left(I_{2 n}\right)^{q}$ consisting of $\left(y_{1}, \cdots, y_{q}\right) q$-tuples of $I_{2 n}$ values. The exact definition of $\mathcal{Y}$ will vary. This $\mathcal{Y}$ will be redefined in each Section where this notation is needed.

\section{Analysis of the L-Scheme}

In this Section, we compare, for various values of the $r$ number of rounds of an L-scheme, the $f=\psi_{L}\left(c_{1}^{*}, c_{2}^{*}, \ldots, c_{r}^{*}\right) 2 n$-bit random permutation deduced from $r$ independent perfect random $n$-bit permutations $c_{1}^{*}, c_{2}^{*}, \ldots, c_{r}^{*}$ with a perfect $2 n$-bit function $f^{*}$ or a $2 n$-bit perfect permutation $c^{*}$. 


\subsection{Three-Round L-Scheme: $\psi_{L}\left(c_{1}^{*}, c_{2}^{*}, c_{3}^{*}\right)$ Is Not a Pseudo-Random Function}

As already noticed by several authors [Zh89], the function $f=\psi_{L}\left(c_{1}^{*}, c_{2}^{*}, c_{3}^{*}\right)$ associated with the three-round L-scheme is not pseudo-random.

Since the omission of the final XOR and the final exchange of the left and right output halves does not affect the pseudorandomness properties of $f$, we can consider the function $f=\psi_{L}^{\prime}\left(c_{1}^{*}, c_{2}^{*}, c_{3}^{*}\right)$, instead of $\psi_{L}\left(c_{1}^{*}, c_{2}^{*}, c_{3}^{*}\right)$.

Let us show that 4 chosen input queries suffice to distinguish $f$ from a the perfect random function $f^{*}$ with a very large probability. Let us consider the encryption, under $f$, of two distinct $2 n$-bit $\left(x^{1}, x^{0}\right)$ plaintext blocks $(a, b)$ and $\left(a^{\prime}, b\right)$ which right halves are equal, and denote by $(c, d)$ and $\left(c^{\prime}, d^{\prime}\right)$ the two corresponding $\left(y^{1}, y^{0}\right)$ ciphertext blocks. We can notice that $d \oplus d^{\prime}$ is equal to $c_{1}^{*}(a) \oplus c_{1}^{*}\left(a^{\prime}\right)$, and thus independent of $b$. Therefore if we replace $b$ by any other value $b^{\prime}$ and do the same computation as before, the new obtained value of $d \oplus d^{\prime}$ will be left unchanged. This property allows to distinguish $f$ from a perfect random function of $I_{2 n}$ with an advantage close to 1 .

\subsection{Four-Round L-Scheme: $\psi_{L}\left(c_{1}^{*}, c_{2}^{*}, c_{3}^{*}, c_{4}^{*}\right)$ Is a Pseudo-Random Function}

As already established by Sakurai et al. [Sa97], the four-round version of the $\mathrm{L}$-scheme is indistinguishable from a perfect pseudo-random function. In order for this paper to provide a self contained summary of the properties of the $\mathrm{L}$ and $\mathrm{R}$ schemes inside the security framework introduced in Section 2, we restate this result as follows.

Theorem 2 Let $n$ be an integer, $c_{1}^{*}, c_{2}^{*}, c_{3}^{*}, c_{4}^{*}$ be four independent random function from $I_{n}$ to $I_{n}$ and $f^{*}$ be the perfect random function on the $I_{2 n}$ set. Let $f=\psi_{L}\left(f_{1}^{*}, f_{2}^{*}, f_{3}^{*}, f_{4}^{*}\right)$ denote the random permutation associated with the four rounds of L-scheme. For any adaptative distinguisher $\mathcal{A}$ with q queries we have:

$$
A d v_{\mathcal{A}}^{q}\left(f, f^{*}\right) \leq \frac{7}{2} q^{2} 2^{-n}
$$

A short proof for Theorem 2 is provided in appendix at the end of this paper. Since the proof technique is rather similar to the one used in the more detailed proof of Theorem 5 on the pseudorandomness of the 3-round R-scheme, we omitted some details in the proof of Theorem 2 .

\subsection{Four-Round L-Scheme: $\psi_{L}\left(c_{1}^{*}, c_{2}^{*}, c_{3}^{*}, c_{4}^{*}\right)$ Is Not a Super Pseudo-Random Permutation}

As already established by Sakurai and al. [Sa97], the 4-round L-scheme does not provide a super pseudo random permutation, i.e. it is possible with a small number of encryption and decryption queries to distinguish $\psi_{L}\left(c_{1}^{*}, c_{2}^{*}, c_{3}^{*}, c_{4}^{*}\right)$ from a perfect random permutation. 
Instead of providing here a direct proof of this property, let us show that this is a straightforward consequence of the fact (established in the next Section) that the 4-round R-scheme does not provide a super pseudo random function. As a matter of fact, $\psi_{R}^{\prime}\left(c_{1}, c_{2}, c_{3}, c_{4}\right)$ and $\psi_{L}^{\prime}\left(c_{4}^{-1}, c_{3}^{-1}, c_{2}^{-1}, c_{1}^{-1}\right)$ are inverse of each other, as stated in Section 2 and therefore, the distinguisher for $\psi_{R}\left(c_{1}^{*}, c_{2}^{*}, c_{3}^{*}, c_{4}^{*}\right)$ can be converted in a distinguisher for $\psi_{L}\left(c_{1}^{*}, c_{2}^{*}, c_{3}^{*}, c_{4}^{*}\right)$ with the same number of queries and the same advantage.

\subsection{Five-Round L-Scheme: $\psi_{L}\left(c_{1}^{*}, c_{2}^{*}, c_{3}^{*}, c_{4}^{*}, c_{5}^{*}\right)$ Is a Super Pseudo-Random Permutation}

Recall that a super pseudo random distinguisher is an adaptative distinguisher which can call at one and the same time the cipher $c$ and the cipher $c^{-1}$. The following Theorem shows that the five-round version of the L-scheme provides a super pseudorandom permutation.

Theorem 3 Let $n$ be an integer, $c_{1}^{*}, c_{2}^{*}, c_{3}^{*}, c_{4}^{*}, c_{5}^{*}$ be five independent random functions from $I_{n}$ to $I_{n}$ and $c^{*}$ be the perfect random permutation on the $I_{2 n}$ set. Let $c=\psi_{L}\left(c_{1}^{*}, c_{2}^{*}, c_{3}^{*}, c_{4}^{*}, c_{5}^{*}\right)$ denote the random permutation associated with the five round $L$ scheme. For any adaptative super pseudorandom permutation distinguisher $\mathcal{A}$ with q queries, we have:

$$
A d v_{\mathcal{A}}^{q}\left(c, c^{*}\right) \leq \frac{9}{2} \cdot \frac{q^{2}}{2^{n}}
$$

To prove this theorem, we need to use a variant of Theorem 1 due to Patarin in [Pa91] concerning permutations (that we provide here without proof):

Theorem 4 Let $m$ be an integer, $\epsilon$ be a positive real number, $c$ be a random permutation on the $\{0,1\}^{m}$ set, $c^{*}$ be the perfect random permutation on the same set. We denote by $\mathcal{X}$ the subset of $\left(X_{1}, \cdots, X_{q}\right)$ q-tuples that are pairwise distinct. Let $\mathcal{A}$ be any super pseudo random distinguisher with q queries. If $\operatorname{Pr}[X \stackrel{c}{\mapsto} Y]$ $\geq(1-\epsilon) \cdot \frac{1}{\left|I_{m}\right|^{q}}$ for all $X$ and $Y$-tuples in $\mathcal{X}$ then $A d v_{\mathcal{A}}^{q}\left(c, c^{*}\right) \leq \epsilon+\frac{q(q-1)}{2 \cdot 2^{m}}$

Proof of Theorem 3: We will compare the $c=\psi_{L}^{\prime}\left(c_{1}^{*}, c_{2}^{*}, c_{3}^{*}, c_{4}^{*}, c_{5}^{*}\right)$ permutation generator of Figure 2 (wich superpseudorandomness properties are exactly the same as for $\left.\psi_{L}\left(c_{1}^{*}, c_{2}^{*}, c_{3}^{*}, c_{4}^{*}, c_{5}^{*}\right)\right)$ with the perfect random permutation $c^{*}$ of $I_{2 n}$. For that purpose, let us consider any $X=\left(x_{i}^{1}, x_{i}^{0}\right) \in \mathcal{X}$ q-tuple of pairwise distinct values of $I_{2 n}$ and any $Y=\left(y_{i}^{1}, y_{i}^{0}\right)$ q-tuple of pairwise distinct values of $I_{2 n}$. We want to establish lower bound on $\operatorname{Pr}[X \stackrel{c}{\mapsto} Y]$ and then apply Theorem 4 above. We are using the notation $x^{2}=\left(x_{i}^{2}\right)_{i=1 . . q}, x^{3}=\left(x_{i}^{3}\right)_{i=1 . . q}, x^{4}=\left(x_{i}^{4}\right)_{i=1 . . q}$ to refer to the q-tuples of $I_{n}$ intermediate words induced by the $q$ considered $f$ computations, at the locations marked in Figure 2.

$$
\begin{aligned}
& \operatorname{Pr}[X \stackrel{c}{\mapsto} Y]=\sum_{x^{2}, x^{3}, x^{4}} \operatorname{Pr}\left[\left(c_{1}^{*}\left(x^{1}\right) \oplus x^{0}=x^{2}\right) \wedge\left(c_{2}^{*}\left(x^{0}\right) \oplus x^{2}=x^{3}\right)\right. \\
& \wedge\left(c_{3}^{*}\left(x^{2}\right) \oplus x^{3}=x^{4}\right)
\end{aligned}
$$




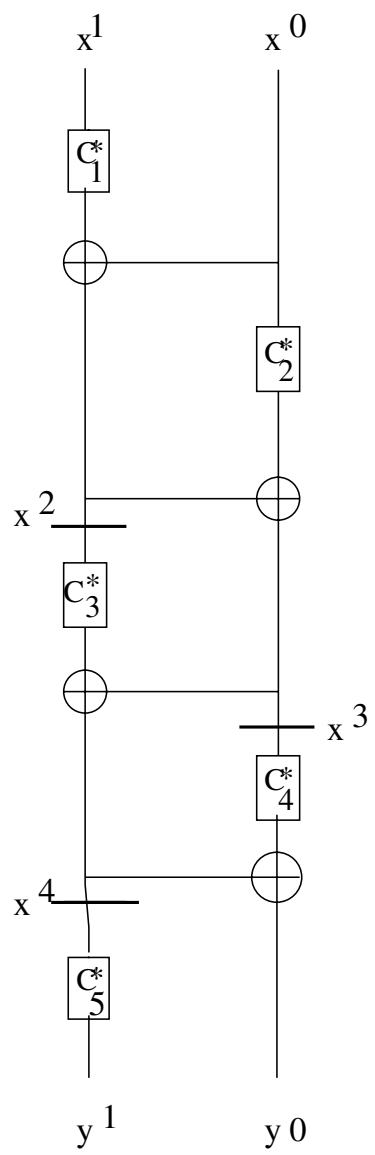

Fig. 2. L-scheme five rounds

$$
\begin{gathered}
\left.\wedge\left(c_{4}^{*}\left(x^{3}\right) \oplus x^{4}=y^{0}\right) \wedge\left(c_{5}^{*}\left(x^{4}\right)=y^{1}\right)\right] \\
\geq \sum_{x^{2}, x^{3} \in I^{\neq}} \operatorname{Pr}\left[\left(c_{1}^{*}\left(x^{1}\right) \oplus x^{0}=x^{2}\right) \wedge\left(c_{2}^{*}\left(x^{0}\right) \oplus x^{2}=x^{3}\right)\right] \\
\cdot \sum_{x^{4}} \quad \operatorname{Pr}\left[\begin{array}{c}
\left(c_{3}^{*}\left(x^{2}\right) \oplus x^{3}=x^{4}\right) \wedge \\
\left(c_{4}^{*}\left(x^{3}\right) \oplus x^{4}=y^{0}\right) \wedge\left(c_{5}^{*}\left(x^{4}\right)=y^{1}\right)
\end{array}\right]
\end{gathered}
$$

Let us consider any fixed $x^{2}, x^{3}$ q-tuples of $I^{\neq}$. In order to establish a lower bound on the $\sum_{x^{4}} \operatorname{Pr}\left[\left(c_{3}^{*}\left(x^{2}\right) \oplus x^{3}=x^{4}\right) \wedge\left(c_{4}^{*}\left(x^{3}\right) \oplus x^{4}=y^{0}\right) \wedge\left(c_{5}^{*}\left(x^{4}\right)=y^{1}\right)\right]$ factor in (2), we define the following set $Z$ of $x^{4}$ q-tuples:

$$
Z=\left\{x^{4} \mid x^{4} \sim y^{1} \wedge x^{4} \oplus y^{0} \in I^{\not} \wedge x^{4} \oplus x^{3} \in I^{\neq}\right\}
$$

where $x^{4} \sim y^{1}$ means that $\forall i, j x_{i}^{4}=x_{j}^{4}$ if and only if $y_{i}^{1}=y_{j}^{1}$. Let us denote by $q_{1} \leq q$, the number of distinct $y_{i}^{1}$ values. there exist $i_{1}, \cdots, i_{q_{1}}$ indexes such that $y_{i_{1}}^{1}, \cdots, y_{i_{q_{1}}}^{1}$ are pairwise distinct. Each $i_{k} \in\left\{i_{1}, \cdots, i_{q_{1}}\right\}$ index determine a class such that for all elements $i$ of this class, $y_{i}^{1}=y_{i_{k}}^{1}$. So, $\forall i \in[1, . ., q], \exists ! i_{k} \in$ $\left\{i_{1}, \cdots, i_{q_{1}}\right\} / y_{i}^{1}=y_{i_{k}}^{1}, C l(i)={ }_{\text {def }} i_{k}$. 
There exist $\alpha=\frac{2^{n} !}{\left(2^{n}-q_{1}\right) !} x^{4}$ values such that $x^{4} \sim y^{1}$ (as a matter of fact such an $x^{4}$ is entirely determined by $q_{1}$ distinct values). Now:

$$
\begin{aligned}
|Z| \geq & \left|\left\{x^{4} \mid x^{4} \sim y^{1}\right\}\right|-\left|\left\{x^{4} \mid x^{4} \sim y^{1} \wedge x^{4} \oplus y^{0} \notin I^{\neq}\right\}\right| \\
& -\left|\left\{x^{4} \mid x^{4} \sim y^{1} \wedge x^{4} \oplus x^{3} \notin I^{\neq}\right\}\right| \\
\geq & \left|\left\{x^{4} \mid x^{4} \sim y^{1}\right\}\right|-\sum_{i \neq j}\left|\left\{x^{4} \mid x^{4} \sim y^{1} \wedge x_{i}^{4} \oplus x_{j}^{4}=y_{i}^{0} \oplus y_{j}^{0}\right\}\right| \\
& -\sum_{i \neq j}\left|\left\{x^{4} \mid x^{4} \sim y^{1} \wedge x_{i}^{4} \oplus x_{j}^{4}=x_{i}^{3} \oplus x_{j}^{3}\right\}\right|
\end{aligned}
$$

Given $i \neq j$, we can upper bound the size of $S_{i j}=\left\{x^{4} \mid x^{4} \sim y^{1} \wedge x_{i}^{4} \oplus x_{j}^{4}=y_{i}^{0} \oplus y_{j}^{0}\right\}$ by $\frac{2 \alpha}{2^{n}}$.

As a matter of fact:

- if $y_{i}^{1}=y_{j}^{1}$, then $y_{i}^{0} \oplus y_{j}^{0} \neq 0$ (because otherwise the $\left(y_{i}^{1}, y_{i}^{0}\right)$ word would be equal to $\left.\left(y_{j}^{1}, y_{j}^{0}\right)\right)$, but $x^{4} \sim y^{1}$ implies $x_{i}^{4}=x_{j}^{4}$ and thus $x_{i}^{4} \oplus x_{j}^{4}$ cannot be equal to $y_{i}^{0} \oplus y_{j}^{0}$. So, $\left|S_{i j}\right|=0$

$-y_{i}^{1} \neq y_{j}^{1}$, then if $x^{4} \in S_{i j}, x_{C l(j)}^{4}$ is entirely determined by $x_{C l(i)}^{4}$ since $x_{C l(j)}^{4}=$ $x_{C l(i)}^{4} \oplus y_{i}^{0} \oplus y_{j}^{0}$. Thus $\left|S_{i j}\right|$ contains at most $2^{n}\left(2^{n}-2\right) \cdots\left(2^{n}-q_{1}\right)=\frac{\alpha}{2^{n}-1} \leq$ $\frac{2 \alpha}{2^{n}}$ elements.

Similary, using the fact that $x_{i}^{3} \neq x_{j}^{3}$, we can upper bound the size of $\left\{x^{4} \mid x^{4} \sim\right.$ $\left.y^{1} \wedge x_{i}^{4} \oplus x_{j}^{4}=x_{i}^{3} \oplus x_{j}^{3}\right\} \mid$ by $\frac{2 \alpha}{2^{n}}$. So, we have:

$$
|Z| \geq \alpha\left[1-\frac{q(q-1)}{2} \frac{2}{2^{n}}-\frac{q(q-1)}{2} \frac{2}{2^{n}}\right] \text { i.e. }|Z| \geq \frac{2^{n} !}{\left(2^{n}-q_{1}\right) !}\left[1-\frac{2 q^{2}}{2^{n}}\right]
$$

Now, $\sum_{x^{4}} \operatorname{Pr}\left[\left(c_{3}^{*}\left(x^{2}\right) \oplus x^{3}=x^{4}\right) \wedge\left(c_{4}^{*}\left(x^{3}\right) \oplus x^{4}=y^{0}\right) \wedge\left(c_{5}^{*}\left(x^{4}\right)=y^{1}\right)\right] \geq$ $\sum_{x^{4} \in Z} \operatorname{Pr}\left[\left(c_{3}^{*}\left(x^{2}\right) \oplus x^{3}=x^{4}\right) \wedge\left(c_{4}^{*}\left(x^{3}\right) \oplus x^{4}=y^{0}\right) \wedge\left(c_{5}^{*}\left(x^{4}\right)=y^{1}\right)\right]$. But, for any $x^{4} \in Z$, we have $\operatorname{Pr}\left[c_{5}^{*}\left(x^{4}\right)=y^{1}\right]=\frac{\left(2^{n}-q_{1}\right) !}{2^{n} !}=\frac{1}{\alpha}$ and $\operatorname{Pr}\left[\left(c_{3}^{*}\left(x^{2}\right)=x^{4} \oplus x^{3}\right)\right]=$ $\frac{\left(2^{n}-q\right) !}{2^{n} !}$ due to Property 2 and the fact that the $x_{i}^{2}$ and the $x_{i}^{4} \oplus x_{i}^{3}$ are pairwise distinct. We also have $\operatorname{Pr}\left[\left(c_{3}^{*}\left(x^{2}\right)=x^{4} \oplus x^{3}\right)\right]=\frac{\left(2^{n}-q\right) !}{2^{n} !}$ for the same reasons, so that:

$\sum_{x^{4}} \operatorname{Pr}\left[\left(c_{3}^{*}\left(x^{2}\right) \oplus x^{3}=x^{4}\right) \wedge\left(c_{4}^{*}\left(x^{3}\right) \oplus x^{4}=y^{0}\right) \wedge\left(c_{5}^{*}\left(x^{4}\right)=y^{1}\right)\right]$

$$
\begin{aligned}
& \geq|Z| \cdot\left(\frac{\left(2^{n}-q\right) !}{2^{n !}}\right)^{2} \cdot \frac{1}{\alpha} \\
& \geq \alpha \cdot\left(1-\frac{2 q^{2}}{2^{n}}\right)\left(\frac{\left(2^{n}-q\right) !}{2^{n} !}\right)^{2} \cdot \frac{1}{\alpha} \\
& \geq\left(1-\frac{2 q^{2}}{2^{n}}\right)\left(\frac{\left(2^{n}-q\right) !}{2^{n} !}\right)^{2}
\end{aligned}
$$


If we now come back to inequality (2), we thus have:

$$
\operatorname{Pr}[X \stackrel{c}{\mapsto} Y] \geq \sum_{x^{2}, x^{3} \in I^{\neq}} \operatorname{Pr}\left[\begin{array}{c}
\left(c_{1}^{*}\left(x^{1}\right) \oplus x^{0}=x^{2}\right) \\
\wedge \\
\left(c_{2}^{*}\left(x^{0}\right) \oplus x^{2}=x^{3}\right)
\end{array}\right] \cdot\left(1-\frac{2 q^{2}}{2^{n}}\right)\left(\frac{\left(2^{n}-q\right) !}{2^{n} !}\right)^{2}
$$

Let us now establish a lower bound on

$$
\begin{aligned}
B= & \sum_{x^{2}, x^{3} \in I^{\neq}} \operatorname{Pr}\left[\left(c_{1}^{*}\left(x^{1}\right) \oplus x^{0}=x^{2}\right) \wedge\left(c_{2}^{*}\left(x^{0}\right) \oplus x^{2}=x^{3}\right)\right] \\
= & \operatorname{Pr}\left[x^{2} \in I^{\neq} \wedge x^{3} \in I^{\neq}\right] \\
& \cdot \operatorname{Pr}\left[\left(c_{1}^{*}\left(x^{1}\right) \oplus x^{0}\right) \in I^{\neq} \wedge\left(c_{2}^{*}\left(x^{0}\right) \oplus x^{2}\right) \in I^{\neq} \mid x^{2} \in I^{\neq}\right]
\end{aligned}
$$

But $\operatorname{Pr}\left[\left(c_{1}^{*}\left(x^{1}\right) \oplus x^{0}\right) \in I^{\neq}\right] \geq 1-\sum_{i \neq j} \operatorname{Pr}\left[c_{1}^{*}\left(x_{i}^{1}\right) \oplus c_{1}^{*}\left(x_{j}^{1}\right)=x_{i}^{0} \oplus x_{j}^{0}\right]$ and it is easy to establish (using the fact that $\left(x_{i}^{1}, x_{i}^{0}\right) \neq\left(x_{j}^{1}, x_{j}^{0}\right)$ and Property 3 ), that for any two distinct indexes $i$ and $j, \operatorname{Pr}\left[c_{1}^{*}\left(x_{i}^{1}\right) \oplus c_{1}^{*}\left(x_{j}^{1}\right)=x_{i}^{0} \oplus x_{j}^{0}\right] \leq \frac{1}{2^{n}-1} \leq \frac{2}{2^{n}}$ Thus $\operatorname{Pr}\left[\left(c_{1}^{*}\left(x^{1}\right) \oplus x^{0}\right) \in I^{\neq}\right] \geq 1-\frac{q(q-1)}{2} \cdot \frac{2}{2^{n}} \geq 1-\frac{q^{2}}{2^{n}}$. for similar reasons, $\operatorname{Pr}\left[\left(c_{2}^{*}\left(x^{0}\right) \oplus x^{2}\right) \in I^{\neq} \mid x^{2} \in I^{\neq}\right] \geq 1-\frac{q^{2}}{2^{n}}$. Thus $B \geq\left(1-\frac{q^{2}}{2^{n}}\right)^{2} \geq 1-\frac{2 q^{2}}{2^{n}}$ (ii) .

Now, by combinig (i) and (ii), we obtain:

$$
\operatorname{Pr}[X \stackrel{c}{\mapsto} Y] \geq\left(1-\frac{2 q^{2}}{2^{n}}\right)^{2}\left(\frac{\left(2^{n}-q\right) !}{2^{n} !}\right)^{2}
$$

Now, $\left(\frac{\left(2^{n}-q\right) !}{2^{n !}}\right)^{2} \geq \frac{1}{2^{2 n q}}$ and $\left(1-\frac{2 q^{2}}{2^{n}}\right)^{2} \geq 1-\frac{4 q^{2}}{2^{n}}$, so that

$$
\operatorname{Pr}[X \stackrel{c}{\mapsto} Y] \geq\left(1-\frac{4 q^{2}}{2^{n}}\right) \cdot \frac{1}{2^{2 n q}}
$$

We can now apply Theorem 4 with $\epsilon=\frac{4 q^{2}}{2^{n}}$ and we obtain:

$$
\begin{gathered}
\operatorname{Adv}_{\mathcal{A}}^{q}\left(c, c^{*}\right) \leq \frac{4 q^{2}}{2^{n}}+\frac{q(q-1)}{2 \cdot 2^{2 n}} \\
\operatorname{Adv}_{\mathcal{A}}^{q}\left(c, c^{*}\right) \leq \frac{9}{2} \cdot \frac{q^{2}}{2^{n}}
\end{gathered}
$$

\section{Analysis of the R-Scheme}

In this Section, we compare, for various values of the $r$ number of rounds of an R-scheme, the $f=\psi_{R}\left(c_{1}^{*}, c_{2}^{*}, \ldots, c_{r}^{*}\right) 2 n$-bit random permutation deduced from $r$ independent perfect random $n$-bit permutations $c_{1}^{*}, c_{2}^{*}, \ldots, c_{r}^{*}$ with a perfect $2 n$-bit function $f^{*}$. 


\subsection{Three-Round R-Scheme}

We first establish the following theorem for a 3-round version of the R-scheme.

Theorem 5 Let $n$ be an integer, $c_{1}^{*}, c_{2}^{*}, c_{3}^{*}$ be three independent perfect random permutation from $I_{n}$ to $I_{n}$ and $f^{*}$ be the perfect random function on the $I_{2 n}$ set. Let $f=\psi_{R}\left(c_{1}^{*}, c_{2}^{*}, c_{3}^{*}\right)$ denote the random permutation associated with the 3-rounds $R$-scheme. For any adaptive distinguisher $\mathcal{A}$ with q queries, we have:

$$
A d v_{\mathcal{A}}^{q}\left(f, f^{*}\right) \leq 3 q^{2} 2^{-n}
$$

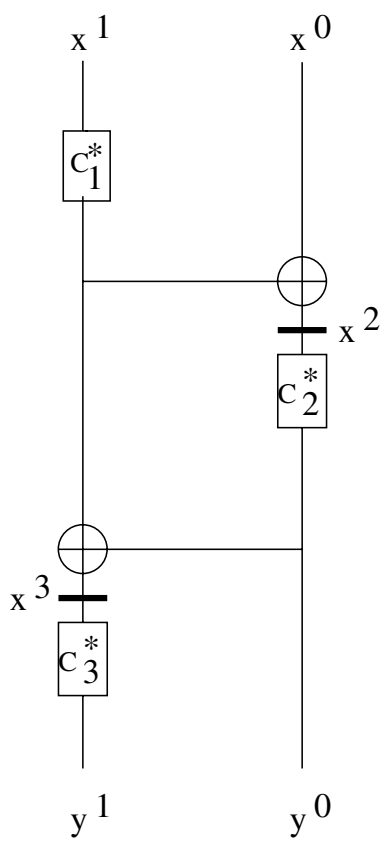

Fig. 3. R-scheme three rounds

Proof: We will compare the $f=\psi_{R}^{\prime}\left(c_{1}^{*}, c_{2}^{*}, c_{3}^{*}\right)$ permutation generator of Figure 3 (which pseudorandomness properties are exactly the same as for $\psi_{R}\left(c_{1}^{*}, c_{2}^{*}, c_{3}^{*}\right)$ ) with the perfect random function $f^{*}$. Let us first introduce some notation. We consider a $X=\left(X_{i}\right)_{i \in[1 . . q]}=\left(x_{i}^{1}, x_{i}^{0}\right) q$-tuple of $2 n$-bit $f$ input words. We denote the corresponding $q$-tuple of $f$ output words by $Y=\left(Y_{i}\right)_{i \in[1 . . q]}=\left(y_{i}^{1}, y_{i}^{0}\right)_{i \in[1 . . q]}$. For each $\left(y_{i}^{1}, y_{i}^{0}\right)=\psi_{R}^{\prime}\left(c_{1}^{*}, c_{2}^{*}, c_{3}^{*}\right)\left(x_{i}^{1}, x_{i}^{0}\right)$ computation, we denote by $x_{i}^{2}$ and $x_{i}^{3}$ the intermediate values which locations are marked in Figure 3. More explicitly:

$$
\begin{gathered}
x_{i}^{2}=c_{1}^{*}\left(x_{i}^{1}\right) \oplus x_{i}^{0} \\
x_{i}^{3}=c_{1}^{*}\left(x_{i}^{1}\right) \oplus c_{2}^{*}\left(x_{i}^{2}\right)=y_{i}^{0} \oplus c_{1}^{*}\left(x_{i}^{1}\right)
\end{gathered}
$$


Finally, we denote the $\left(x_{i}^{0}\right)_{i \in[1 . . q]}, \quad\left(x_{i}^{1}\right)_{i \in[1 . . q]}, \quad\left(x_{i}^{2}\right)_{i \in[1 . . q]}, \quad\left(x_{i}^{3}\right)_{i \in[1 . . q]}$, $\left(y_{i}^{0}\right)_{i \in[1 . . q]}$ and $\left(y_{i}^{1}\right)_{i \in[1 . . q]} q$-tuples of $n$-bit words by $x^{0}, x^{1}, x^{2}, x^{3}, y^{0}, y^{1}$ respectively.

We now define $\mathcal{X}$ as the set of $X$ q-tuples of pairwise distinct $I_{2 n}$ words (i.e. such that for any distinct i,j numbers in $[1 . . q], x_{i}^{1} \neq x_{j}^{1}$ or $x_{i}^{0} \neq x_{j}^{0}$ ), and define $\mathcal{Y}$ as the set of those $Y$ q-tuples of $I_{2 n}$ words such that the corresponding $y^{1}$ and $y^{0}$ q-tuples both consist of pairwise distinct $I_{n}$ words: $\mathcal{Y}=\left\{\left(Y_{1}, \cdots, Y_{q}\right) \in\right.$ $\left.\left(I_{2 n}\right)^{q} / y^{1} \in I^{\neq}, y^{0} \in I^{\neq}\right\}$.

We want to establish a lower bound on the size of $\mathcal{Y}$ and the $\operatorname{Pr}[X \stackrel{f}{\mapsto} Y]$ transition probability associated with any $X$ q-tuple in $\mathcal{X}$ and any $Y$ q-tuple in $\mathcal{Y}$ and show that there exists $\epsilon_{1}$ and $\epsilon_{2}$ real numbers satisfying conditions of Theorem 1.

Let us first establish a lower bound on $|\mathcal{Y}|$. We have:

$$
\begin{aligned}
|\mathcal{Y}| & =\left|I^{2 n}\right|^{q} \cdot\left(1-\operatorname{Pr}\left[y^{1} \notin I^{\neq} \vee y^{0} \notin I^{\ddagger}\right]\right) \\
& \geq\left|I^{2 n}\right|^{q}\left(1-\sum_{i, j \in[1 . . q], i \neq j} \operatorname{Pr}\left[y_{i}^{1}=y_{j}^{1}\right]-\sum_{i, j \in[1 . . q], i \neq j} \operatorname{Pr}\left[y_{i}^{0}=y_{j}^{0}\right]\right) \\
& \geq\left|I^{2 n}\right|^{q}\left(1-\frac{q(q-1)}{2} \cdot 2^{-n}-\frac{q(q-1)}{2} \cdot 2^{-n}\right) \\
& \geq\left|I^{2 n}\right|^{q}\left(1-\frac{q(q-1)}{2^{n}}\right)
\end{aligned}
$$

So, we can take $\epsilon_{1}=\frac{q(q-1)}{2^{n}}$.

Now, given any $X$ q-tuple of $\mathcal{X}$ and any $Y$-tuple of $\mathcal{Y}$ let us establish a lower bound on $\operatorname{Pr}[X \stackrel{f}{\mapsto} Y]$.

$$
\begin{aligned}
\operatorname{Pr}[X \stackrel{f}{\mapsto} Y]=\sum_{x^{2}, x^{3} \in I} & \operatorname{Pr}\left[\left(c_{1}^{*}\left(x^{1}\right) \oplus x^{0}=x^{2}\right) \wedge\left(c_{1}^{*}\left(x^{1}\right) \oplus y^{0}=x^{3}\right)\right. \\
& \left.\wedge\left(c_{2}^{*}\left(x^{2}\right)=y^{0}\right) \wedge\left(c_{3}^{*}\left(x^{3}\right)=y^{1}\right)\right] \\
\geq \sum_{x^{2}, x^{3} \in I^{\neq}} & \operatorname{Pr}\left[\left(c_{1}^{*}\left(x^{1}\right) \oplus x^{0}=x^{2}\right) \wedge\left(c_{1}^{*}\left(x^{1}\right) \oplus y^{0}=x^{3}\right)\right] \\
& \cdot \operatorname{Pr}\left[\left(c_{2}^{*}\left(x^{2}\right)=y^{0}\right) \wedge\left(c_{3}^{*}\left(x^{3}\right)=y^{1}\right)\right]
\end{aligned}
$$

First, for any $x^{2} q$-tuple of $I^{\neq}$and any $x^{3} q$-tuple of $I^{\neq}$, let us compute $\operatorname{Pr}\left[\left(c_{2}^{*}\left(x^{2}\right)=y^{0}\right) \wedge\left(c_{3}^{*}\left(x^{3}\right)=y^{1}\right)\right]=\operatorname{Pr}\left[\left(c_{2}^{*}\left(x^{2}\right)=y^{0}\right)\right] \cdot \operatorname{Pr}\left[\left(c_{3}^{*}\left(x^{3}\right)=y^{1}\right)\right]$. Since $x^{2}$ and $y^{0}$ both belong to $I^{\neq}$, we can can apply Property 2 of Section 2 concerning random permutations, so that $\operatorname{Pr}\left[\left(c_{2}^{*}\left(x^{2}\right)=y^{0}\right)\right]=\frac{\left(2^{n}-q\right) !}{2^{n !}}$. For the same reason, we also have $\operatorname{Pr}\left[\left(c_{3}^{*}\left(x^{3}\right)=y^{1}\right)\right]=\frac{\left(2^{n}-q\right) !}{2^{n} !}$.

So, we have $\operatorname{Pr}\left[\left(c_{2}^{*}\left(x^{2}\right)=y^{0}\right) \wedge\left(c_{3}^{*}\left(x^{3}\right)=y^{1}\right)\right]=\left(\frac{\left(2^{n}-q\right) !}{2^{n !}}\right)^{2}$.

Now, $\left(\frac{\left(2^{n}-q\right) !}{2^{n} !}\right)^{2} \geq \frac{1}{2^{2 n q}}$.

Therefore, inequality (2) implies:

$$
\operatorname{Pr}[X \stackrel{f}{\mapsto} Y] \geq \sum_{x^{2}, x^{3} \in I^{\neq}} \frac{1}{2^{2 n q}} \cdot \operatorname{Pr}\left[\left(c_{1}^{*}\left(x^{1}\right) \oplus x^{0}=x^{2}\right) \wedge\left(c_{1}^{*}\left(x^{1}\right) \oplus y^{0}=x^{3}\right)\right]
$$


Let us now estimate $B=\sum_{x^{2}, x^{3} \in I^{\prime}} \operatorname{Pr}\left[\left(c_{1}^{*}\left(x^{1}\right) \oplus x^{0}=x^{2}\right) \wedge\left(c_{1}^{*}\left(x^{1}\right) \oplus y^{0}=x^{3}\right)\right]$

We have:

$$
\begin{aligned}
B & =\operatorname{Pr}\left[\left(c_{1}^{*}\left(x^{1}\right) \oplus x^{0} \in I^{\neq} \wedge\left(c_{1}^{*}\left(x^{1}\right) \oplus y^{0} \in I^{\neq}\right]\right.\right. \\
= & -\operatorname{Pr}\left[\left(c_{1}^{*}\left(x^{1}\right) \oplus x^{0} \notin I^{\neq} \vee\left(c_{1}^{*}\left(x^{1}\right) \oplus y^{0} \notin I^{\neq}\right]\right.\right. \\
\geq 1- & \sum_{i, j, i \neq j} \operatorname{Pr}\left[c_{1}^{*}\left(x_{i}^{1}\right) \oplus x_{i}^{0}=c_{1}^{*}\left(x_{j}^{1}\right) \oplus x_{j}^{0}\right] \\
& \quad-\sum_{i, j, i \neq j} \operatorname{Pr}\left[c_{1}^{*}\left(x_{i}^{1}\right) \oplus y_{i}^{0}=c_{1}^{*}\left(x_{j}^{1}\right) \oplus y_{j}^{0}\right]
\end{aligned}
$$

Let us evaluate $\operatorname{Pr}\left[c_{1}^{*}\left(x_{i}^{1}\right) \oplus x_{i}^{0}=c_{1}^{*}\left(x_{j}^{1}\right) \oplus x_{j}^{0}\right]$ and $\operatorname{Pr}\left[c_{1}^{*}\left(x_{i}^{1}\right) \oplus c_{1}^{*}\left(x_{j}^{1}\right)=x_{i}^{0} \oplus x_{j}^{0}\right]$. Due to Property 3 of Section 2 , if $x_{i}^{1} \neq x_{j}^{1}$ given any fixed difference $\delta$ (here equal to $\left.x_{i}^{0} \oplus x_{j}^{0}\right), \operatorname{Pr}\left[c_{1}^{*}\left(x_{i}^{1}\right) \oplus c_{1}^{*}\left(x_{j}^{1}\right)=\delta\right] \leq \frac{2}{2^{n}}$. On the other hand, if $x_{i}^{1}=x_{j}^{1}$, then $x_{i}^{0} \neq x_{j}^{0}$, so that $\operatorname{Pr}\left[c_{1}^{*}\left(x_{i}^{1}\right) \oplus x_{i}^{0}=c_{1}^{*}\left(x_{j}^{1}\right) \oplus x_{j}^{0}\right]$. In all cases, $\operatorname{Pr}\left[c_{1}^{*}\left(x_{i}^{1}\right) \oplus x_{i}^{0}=\right.$ $\left.c_{1}^{*}\left(x_{j}^{1}\right) \oplus x_{j}^{0}\right] \leq \frac{2}{2^{n}}$. Applying this property to the $\frac{q(q-1)}{2}(i, j), i \neq j$ pairs of $[1 . . q]$ indexes, we obtain $\sum_{i, j} \operatorname{Pr}\left[c_{1}^{*}\left(x_{i}^{1}\right) \oplus x_{i}^{0}=c_{1}^{*}\left(x_{j}^{1}\right) \oplus x_{j}^{0}\right] \leq \frac{q(q-1)}{2^{n}}$. For the same reasons, $\sum_{i, j, i \neq j} \operatorname{Pr}\left[c_{1}^{*}\left(x_{i}^{1}\right) \oplus y_{i}^{0}=c_{1}^{*}\left(x_{j}^{1}\right) \oplus y_{j}^{0}\right] \leq \frac{q(q-1)}{2^{n}}$. Thus:

$$
B \geq 1-\frac{2 q(q-1)}{2^{n}}
$$

By using inequalities (i) and (ii), we obtain:

$$
\operatorname{Pr}[X \stackrel{f}{\mapsto} Y] \geq\left(1-\frac{2 q(q-1)}{2^{n}}\right) \cdot \frac{1}{2^{2 n q}}
$$

We can notice that $\operatorname{Pr}\left[X \stackrel{f^{*}}{\mapsto} Y\right]=\frac{1}{2^{2 n q}}$. So we can apply Theorem 1 with $\epsilon_{1}=\frac{q(q-1)}{\left|I^{n}\right|}$ and $\epsilon_{2}=\frac{2 q(q-1)}{\left|I^{n}\right|}$. We obtain:

$$
\begin{gathered}
\operatorname{Adv}_{\mathcal{A}}^{q}\left(f, f^{*}\right) \leq \frac{3 q(q-1)}{2^{n}} \\
\operatorname{Adv}_{\mathcal{A}}^{q}\left(f, f^{*}\right) \leq \frac{3 q^{2}}{2^{n}}
\end{gathered}
$$

\subsection{Four-Round R-Scheme: $\psi_{R}\left(c_{1}^{*}, c_{2}^{*}, c_{3}^{*}, c_{4}^{*}\right)$ Is Not a Super Pseudo-Random Permutation}

We consider the four-round permutation generator $f$ deduced from $\psi_{R}\left(c_{1}^{*}, c_{2}^{*}, c_{3}^{*}, c_{4}^{*}\right)$ by omitting the final XOR (this does obviously not matter for the super pseudo randomness issue considered here). The random function $f$ can be represented by extending the 3-round function of Figure 3 above by one round.

Let us show that 2 chosen encryption and two chosen decryption queries suffice to distinguish $f$ from a the perfect random permutation $c *$ with a very large probability. Let us consider the encryption, under the function $f$, of two 
distinct $2 n$-bit $\left(x^{1}, x^{0}\right)$ plaintext blocks $(a, b)$ and $\left(a, b^{\prime}\right)$ which left halves are equal, and denote by $(c, d)$ and $\left(c^{\prime}, d^{\prime}\right)$ the two obtained $\left(y^{1}, y^{0}\right)$ ciphertext blocks. It is easy to check that if we swap the left halves of the two obtained ciphertexts, thus obtaining two modified ciphertexts $\left(c^{\prime}, d\right)$ and $\left(c, d^{\prime}\right)$ and if we decrypt $\left(c^{\prime}, d\right)$ and $\left(c, d^{\prime}\right)$ under $f^{-1}$, we obtain two plantext values $\alpha, \beta$ and $\alpha^{\prime}, \beta^{\prime}$ which left halves are equal: $\alpha=\alpha^{\prime}$. This would be extremely unlikely to happen if $f$ was replaced by a perfect random permutation $c *$.

The above test allows to distinguish $f$ from a perfect random permutation of $I_{2 n}$ with a probability close to 1 .

\subsection{Five-Round R-Scheme: $\psi_{R}\left(c_{1}^{*}, c_{2}^{*}, c_{3}^{*}, c_{4}^{*}, c_{5}^{*}\right)$ Is a Super Pseudo-Random Permutation}

The following theorem is a direct consequence of Theorem 3 due to the fact that $\psi_{R}^{\prime}\left(c_{1}, c_{2}, c_{3}, c_{4}, c_{5}\right)$ and $\psi_{L}^{\prime}\left(c_{1}^{-1}, c_{2}^{-1}, c_{3}^{-1}, c_{4}^{-1}, c_{5}^{-1}\right)$ are inverse of each other, every distinguisher for $\psi_{R}\left(c_{1}^{*}, c_{2}^{*}, c_{3}^{*}, c_{4}^{*}, c_{5}^{*}\right)$ can be converted into a distinguisher for $\psi_{L}\left(c_{1}^{*}, c_{2}^{*}, c_{3}^{*}, c_{4}^{*}, c_{5}^{*}\right)$ with the same number of encryption and decryption queries. Therefore, Theorem 3 results in the following analogue theorem for the 5 -round R-scheme.

Theorem 6 Let $n$ be an integer, $c_{1}^{*}, c_{2}^{*}, c_{3}^{*}, c_{4}^{*}, c_{5}^{*}$ be five independent random functions from $I_{n}$ to $I_{n}$ and $c^{*}$ be the perfect random permutation on the $I_{2 n}$ set. Let $c=\psi_{R}\left(c_{1}^{*}, c_{2}^{*}, c_{3}^{*}, c_{4}^{*}, c_{5}^{*}\right)$ denote the random permutation associated with the five round $R$-scheme. For any adaptive super pseudorandom permutation distinguisher $\mathcal{A}$ with q queries, we have:

$$
A d v_{\mathcal{A}}^{q}\left(c, c^{*}\right) \leq \frac{9}{2} \cdot \frac{q^{2}}{2^{n}}
$$

\section{Conclusion}

As a consequence of previous results, the security properties of the L-scheme and the R-scheme are distinct when it comes to chosen plaintext attacks, but equivalent when it comes to chosen plaintext or ciphertext attacks. As a matter of fact, the minimal number of rounds required in order of the R-scheme to be undistinguishable from a pseudorandom function with adaptively chosen encryption queries is less than for the L-scheme (3 rounds instead of 4 ), whereas the minimal numbers of rounds required by the R-scheme and the L-scheme in order to be undistinguishable from a pseudorandom permutation with adaptively chosen encryption or decryption queries are equal (5 rounds for both schemes).

\section{A Appendix}

\section{A.1 A Short Proof of Theorem 1}

Let us restrict ourselves to the case of any fixed deterministic algorithm $A$ which uses $q$ adaptively chosen queries (the generalisation to the case of a probabilistic algorithm is easy). 
$A$ has the property that if the $q$-uple of outputs encountered during an $A$ computation is $Y=\left(Y_{1}, \cdots, Y_{q}\right)$, the value of the $X=\left(X_{1}, \cdots, X_{q}\right) q$-uple $\mathrm{n}$ is of query inputs encountered during this computation is entirely determined (this is easy to prove by induction: the initial query input $X_{1}$ is fixed ; if for a given $A$ computation the first query output is $Y_{1}$, then $X_{2}$ is determined, etc.). We denote by $X(Y)$ the single $q$-uple of query inputs corresponding to any possible $Y q$-uple of query outputs, and we denote by $S_{A}$ the subset of those $Y \in I_{m}{ }^{q}$ values such that if the $X(Y)$ and $Y q$-uples query inputs and outputs are encountered in a $A$ computation, then $A$ outputs a 1 answer.

The $p$ and $p^{*}$ probabilities can be expressed using $S_{A}$ as

$$
p=\sum_{Y \in S_{A}} \operatorname{Pr}[X(Y) \stackrel{f}{\mapsto} Y] \text { and } p^{*}=\sum_{Y \in S_{A}} \operatorname{Pr}\left[X(Y) \stackrel{f^{*}}{\mapsto} Y\right]
$$

We can now lower bound $p$ using the following inequalities:

$p \geq \sum_{Y \in S_{A} \cap \mathcal{Y}}\left(1-\epsilon_{2}\right) \cdot \operatorname{Pr}\left[X(Y) \stackrel{f^{*}}{\mapsto} Y\right] \quad$ (due to inequality (ii))

$\geq \sum_{Y \in S_{A}}\left(1-\epsilon_{2}\right) \operatorname{Pr}\left[X(Y) \stackrel{f^{*}}{\mapsto} Y\right]-\sum_{Y \in I_{m}{ }^{q}{ }_{m} \text { athcal } Y}\left(1-\epsilon_{2}\right) \cdot \operatorname{Pr}\left[X(Y) \stackrel{f^{*}}{\mapsto} Y\right]$ But

$$
\begin{gathered}
\sum_{Y \in S_{A}}\left(1-\epsilon_{2}\right) \cdot \operatorname{Pr}\left[X(Y) \stackrel{f^{*}}{\mapsto} Y\right]=\left(1-\epsilon_{2}\right) p^{*} \\
\quad \text { and } \\
\sum_{Y \in I_{m}{ }^{q}-\mathcal{Y}}\left(1-\epsilon_{2}\right) \cdot \operatorname{Pr}\left[X(Y) \stackrel{f^{*}}{\mapsto} Y\right]=\left(1-\epsilon_{2}\right) \cdot \frac{\left|I_{m}\right|^{q}-|\mathcal{Y}|}{\left|I_{m}\right|} \leq\left(1-\epsilon_{2}\right) \cdot \epsilon_{1} \text { (due to } \\
\text { inequality (i)). }
\end{gathered}
$$

Therefore $p \geq\left(1-\epsilon_{2}\right)\left(p^{*}-\epsilon_{1}\right)=p^{*}-\epsilon_{1}-\epsilon_{2} \cdot p^{*}+\epsilon_{1} \cdot \epsilon_{2}$

thus finally (using $p^{*} \leq 1$ and $\epsilon_{1} \cdot \epsilon_{2} \geq 0$ )

$p \geq p *-\epsilon_{1}-\epsilon_{2} \quad$ (a)

If we now consider the $A^{\prime}$ distinguisher which outputs are the inverse of those of $A$ (i.e. $A^{\prime}$ answers 0 iff $A$ answers 1 ), we obtain an inequality involving this time $1-p$ and $1-p *$ :

$(1-p) \geq(1-p *)-\epsilon_{1}-\epsilon_{2} \quad$ (b)

Combining inequalities (a) and (b), we obtain $|p-p *| \leq \epsilon_{1}+\epsilon_{2}$ QED.

\section{A.2 A Proof Sketch for Theorem 2}

We will compare the $f=\psi_{L}^{\prime}\left(c_{1}^{*}, c_{2}^{*}, c_{3}^{*}, c_{4}^{*}\right)$ permutation generator of Figure 4 (which pseudorandomness properties are exactly the same as for $\left.\psi_{L}\left(c_{1}^{*}, c_{2}^{*}, c_{3}^{*}, c_{4}^{*}\right)\right)$ with the perfect random function $f^{*}$. This proof is near to the proof of Section 5.1. That's why we do not detail some computations that are the same that in Section 5.1.

Let us first introduce some notation. We consider a $X=\left(X_{i}\right)_{i \in[1 . . q]}=\left(x_{i}^{1}, x_{i}^{0}\right)$ q-tuple of 2 n-bit $f$ input words. We denote the corresponding q-tuple of $f$ output words by $Y=\left(Y_{i}\right)_{i \in[1 . . q]}=\left(y_{i}^{1}, y_{i}^{0}\right)_{i \in[1 . . q]}$. For each $\left(y_{i}^{1}, y_{i}^{0}\right)=$ $\psi_{L}^{\prime}\left(c_{1}^{*}, c_{2}^{*}, c_{3}^{*}, c_{4}^{*}\right)\left(x_{i}^{1}, x_{i}^{0}\right)$ computation, we denote by $x_{i}^{2}$ and $x_{i}^{3}$ the intermediate values which locations are marked in Figure 4. More explicitly:

$$
\begin{aligned}
x_{i}^{2} & =c_{1}^{*}\left(x_{i}^{1}\right) \oplus x_{i}^{0} \\
x_{i}^{3} & =c_{2}^{*}\left(x_{i}^{0}\right) \oplus x_{i}^{2}
\end{aligned}
$$




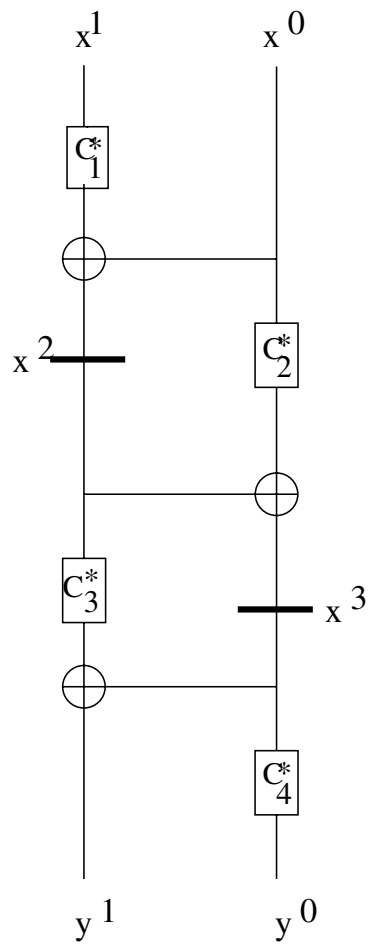

Fig. 4. L-scheme four rounds

Finally, we denote the $\left(x_{i}^{0}\right)_{i \in[1 . . q]}, \quad\left(x_{i}^{1}\right)_{i \in[1 . . q]}, \quad\left(x_{i}^{2}\right)_{i \in[1 . . q]}, \quad\left(x_{i}^{3}\right)_{i \in[1 . . q]}$, $\left(y_{i}^{0}\right)_{i \in[1 . . q]}$ and $\left(y_{i}^{1}\right)_{i \in[1 . . q]}$ q-tuples of n-bit words by $x^{0}, x^{1}, x^{2}, x^{3}, y^{0}, y^{1}$ respectively.

We now define $\mathcal{X}$ as the set of $X$ q-tuples of pairwise distinct $I_{2 n}$ words (i.e. such that for any distinct i,j numbers in [1..q], $x_{i}^{1} \neq x_{j}^{1}$ or $x_{i}^{0} \neq x_{j}^{0}$ ), and define $\mathcal{Y}$ as the set of those $Y$ q-tuples of $I_{2 n}$ words such that the corresponding $y^{1}$ q-tuples consists of pairwise distinct $I_{n}$ words: $\mathcal{Y}=\left\{\left(Y_{1}, \cdots, Y_{q}\right) \in\left(I^{2 n}\right)^{q} / y^{1} \in\right.$ $\left.I^{\neq}, y^{0} \in I^{\neq}\right\}$.

We want to lower bound the size of $\mathcal{Y}$ and the $\operatorname{Pr}[X \stackrel{f}{\mapsto} Y]$ transition probability associated with any $X$ q-tuple in $\mathcal{X}$ and any $Y$ q-tuple in $\mathcal{Y}$ and show that there exists $\epsilon_{1}$ and $\epsilon_{2}$ real numbers satisfying conditions of Theorem 1.

We have (for more details, see section 5.1):

$$
\begin{aligned}
|\mathcal{Y}| & =\left|I^{2 n}\right|^{q} \cdot\left(1-\operatorname{Pr}\left[y^{1} \notin I^{\neq}\right]\right) \\
& \geq\left|I^{2 n}\right|^{q}\left(1-\frac{q(q-1)}{2.2^{n}}\right)
\end{aligned}
$$

So, we can take $\epsilon_{1}=\frac{q(q-1)}{2.2^{n}}$. 
Now, given any $X$ q-tuple of $\mathcal{X}$ and any $Y$ q-tuple of $\mathcal{Y}$ let us establish a lower bound on $\operatorname{Pr}[X \stackrel{f}{\mapsto} Y]$ (for more details, see section 5.1).

$$
\begin{gathered}
\left.\operatorname{Pr}[X \stackrel{f}{\mapsto} Y] \geq \sum_{x^{2}, x^{3}, x^{3} \oplus y^{1} \in I^{\neq}} \operatorname{Pr}\left[\left(c_{1}^{*}\left(x^{1}\right) \oplus x^{0}=x^{2}\right) \wedge\left(x^{3}=c_{2}^{*}\left(x^{0}\right)\right)\right] \oplus x^{2}\right) \\
\cdot \operatorname{Pr}\left[\left(c_{3}^{*}\left(x^{2}\right) \oplus x^{3}=y^{0}\right) \wedge\left(c_{4}^{*}\left(x^{3}\right)=y^{1}\right)\right]
\end{gathered}
$$

First, for any $x^{2}, x^{3}, x^{3} \oplus y^{1}$ q-tuple of $I^{\neq}$, we have $\operatorname{Pr}\left[\left(c_{3}^{*}\left(x^{2}\right) \oplus x^{3}=\right.\right.$ $\left.\left.y^{0}\right) \wedge\left(c_{3}^{*}\left(x^{3}\right)=y^{1}\right)\right]=\left(\frac{\left(2^{n}-q\right) !}{2^{n} !}\right)^{2} \geq \frac{1}{2^{2 n q}}$ (for more details, see section 5.1). Therefore, inequality (1) implies:

$$
\operatorname{Pr}[X \stackrel{f}{\mapsto} Y] \geq \sum_{x^{2}, x^{3}, x^{3} \oplus y^{1} \in I^{\neq}} \frac{1}{2^{2 n q}} \cdot \operatorname{Pr}\left[\left(c_{1}^{*}\left(x^{1}\right) \oplus x^{0}=x^{2}\right) \wedge\left(c_{1}^{*}\left(x^{1}\right) \oplus y^{0}=x^{3}\right)\right]
$$

Let us now estimate:

$$
B=\sum_{x^{2}, x^{3}, x^{3} \oplus y^{1} \in I^{\neq}} \operatorname{Pr}\left[\left(c_{1}^{*}\left(x^{1}\right) \oplus x^{0}=x^{2}\right) \wedge\left(c_{2}^{*}\left(x^{0}\right) \oplus x^{2}=x^{3}\right)\right]
$$

We have:

$$
\begin{aligned}
B & =\operatorname{Pr}\left[\left(c_{1}^{*}\left(x^{1}\right) \oplus x^{0}\right) \in I^{\neq} \wedge\left(c_{2}^{*}\left(x^{0}\right) \oplus c_{1}^{*}\left(x^{1}\right) \oplus x^{0}\right) \in I^{\neq} \wedge\left(x^{3} \oplus y^{1}\right) \in I^{\neq}\right] \\
& =1-\operatorname{Pr}\left[\left(c_{1}^{*}\left(x^{1}\right) \oplus x^{0} \in I^{=}\right) \vee\left(c_{2}^{*}\left(x^{0}\right) \oplus c_{1}^{*}\left(x^{1}\right) \oplus x^{0}\right) \in I^{=} \vee\left(x^{3} \oplus y^{1}\right) \in I^{=}\right] \\
& \geq 1-3 \cdot \frac{q(q-1)}{2} \cdot \frac{2}{2^{n}}
\end{aligned}
$$

By using inequalities (i) and (ii), we obtain:

$$
\operatorname{Pr}[X \stackrel{f}{\mapsto} Y] \geq\left(1-\frac{3 q(q-1)}{2^{n}}\right) \cdot \frac{1}{2^{2 n q}}
$$

We can notice that $\operatorname{Pr}\left[X \stackrel{f^{*}}{\mapsto} Y\right]=\frac{1}{2^{2 n q}}$. So we can apply Theorem 1 with $\epsilon_{1}=\frac{q(q-1)}{2\left|I^{n}\right|}$ and $\epsilon_{2}=\frac{3 q(q-1)}{\left|I^{n}\right|}$. We obtain:

$$
\begin{gathered}
\operatorname{Adv}_{\mathcal{A}}^{q}\left(f, f^{*}\right) \leq \frac{7 q(q-1)}{2.2^{n}} \\
\operatorname{Adv}_{\mathcal{A}}^{q}\left(f, f^{*}\right) \leq \frac{7 q^{2}}{2.2^{n}}
\end{gathered}
$$

\section{References}

[Ai96] W. Aiello, R. Venkatesan, "Foiling Birthday Attacks in Length-Doubling Transformations". In Advances in Cryptology - Eurocrypt'96, LNCS 1070, p. 307, Springer Verlag, Saragossa, Spain, May 1996.

[Be94] M. Bellare, J. Kilian, P. Rogaway, "The Security of Cipher Block Chaining". In Advances in Cryptology - CRYPTO'94,LNCS 839, p. 341, Springer-Verlag, Santa Barbara, U.S.A., 1994. 
[Ka] Specification of the 3GPP confidentiality and Integrity algorithm KASUMI. Documentation available on http://www.etsi.org/

[Ka97] Y. Kaneko, F. Sano, K. Sakurai, "On Provable Security against Differential and Linear Cryptanalysis in Generalized Feistel Ciphers with Multiple Random Functions". In Selected Areas in Cryptography - SAC'97, Ottawa, Canada, August 1997.

[La90] X. Lai, J.L. Massey, "A Proposal for a New Block Encryption Standard". In Advances in Cryptology - Eurocrypt'90, LNCS 473 , p. 389, Springer Verlag, Aarhus, Denemark, 1991.

[Lu88] M. Luby, C. Rackoff, "How to Construct Pseudorandom Permutations from Pseudorandom Function". In Siam Journal on Computing, vol. 17, p. 373, 1988.

[Ma92] U. Maurer, "A Simplified and generalised treatment of Luby-Rackoff Pseudorandom Permutation Generators", In Advances in Cryptology - Eurocrypt'92, LNCS 658 , p. 239, Springer Verlag, New York, USA, 1992.

[Ma93] M. Matsui, "New Block Encryption Algorithm MISTY", In Fast Software Encryption - FSE'97, LNCS 1267, p. 54, Springer Verlag, Haifa, Israel, 1997.

[Pa91] J. Patarin, "Etude de Générateurs de Permutation Basés sur le Schéma du D.E.S. ", Phd. Thesis, University of Paris VI, 1991.

[Sa97] K. Sakurai, Y. Zheng, "On Non-Pseudorandomness from Block Ciphers with Provable Immunity Against Linear Cryptanalysis, In IEICE Trans. Fundamentals, vol. E80-A, n. 1, January 1997.

[Su96] M. Sugita, "Pseudorandomness of a Block Cipher MISTY", Technical Reporrt of IEICE, ISEC96-9.

[Su97] M. Sugita, "Pseudorandomness of a Block Cipher with Recursive Structures", Technical Report of IEICE, ISEC97-9.

[Va99] S. Vaudenay, "On Provable Security for Conventional Cryptography", In ICISC'99, invited lecture.

[Zh89] Y. Zheng, T. Matsumoto, H. Imai, "On the Construction of Block Ciphers Provably Secure and Not Relying on Any Unproved Hypotheses". In $A d$ vances in Cryptology - CRYPTO'89, LNCS 435, p. 461, Springer-Verlag, Santa Barbara, U.S.A., 1990. 